\title{
Utilization of key preventive measures for pregnancy complications and malaria among women in Jimma Zone, Ethiopia
}

Mariame Ouedraogo', Jaameeta Kurji', Lakew Abebe², Ronald Labonté1, Sudhakar Morankar², Kunuz Haji Bedru², Gebeyehu Bulcha ${ }^{3}$, Muluemebet Abera ${ }^{4}$, Beth K. Potter ${ }^{1}$, Marie-Hélène Roy-Gagnon ${ }^{1}$ and Manisha A. Kulkarni ${ }^{{ }^{*}}$ (D)

\begin{abstract}
Background: In Ethiopia, malaria infections and other complications during pregnancy contribute to the high burden of maternal morbidity and mortality. Preventive measures are available, however little is known about the factors influencing the uptake of maternal health services and interventions by pregnant women in Ethiopia.

Methods: We analyzed data from a community-based cross-sectional survey conducted in 2016 in three rural districts of Jimma Zone, Ethiopia, with 3784 women who had a pregnancy outcome in the year preceding the survey. We used multivariable logistic regression models accounting for clustering to identify the determinants of antenatal care (ANC) attendance and insecticide-treated net (ITN) ownership and use, and the prevalence and predictors of malaria infection among pregnant women.

Results: Eighty-four percent of interviewed women reported receiving at least one ANC visit during their last pregnancy, while $47 \%$ reported attending four or more ANC visits. Common reasons for not attending ANC included women's lack of awareness of its importance (48\%), distance to health facility (23\%) and unavailability of transportation (14\%). Important determinants of ANC attendance included higher education level and wealth status, woman's ability to make healthcare decisions, and pregnancy intendedness. An estimated $48 \%$ of women reported owning an ITN during their last pregnancy. Of these, 55\% reported to have always slept under it during their last pregnancy. Analysis revealed that the odds of owning and using ITNs were respectively 2.07 (95\% Cl: 1.62-2.63) and 1.73 (95\% Cl: 1.32-2.27) times higher among women who attended at least one ANC visit. The self-reported prevalence of malaria infection during pregnancy was low (1.4\%) across the three districts. We found that young, uneducated, and unemployed women presented higher odds of malaria infection during their last pregnancy.

Conclusion: ANC and ITN uptake during pregnancy in Jimma Zone fall below the respective targets of 95 and $90 \%$ set in the Ethiopian Health Sector Transformation Plan for 2020, suggesting that more intensive programmatic efforts still need to be directed towards improving access to these health services. Reaching ANC non-users and ITN ownership and use as part of ANC services could be emphasized to address these gaps.
\end{abstract}

Keywords: Pregnant women, Antenatal care, Insecticide-treated nets, Malaria, Ethiopia

\footnotetext{
* Correspondence: manisha.kulkarni@uottawa.ca

${ }^{1}$ School of Epidemiology and Public Health, Faculty of Medicine, University

of Ottawa, Ottawa, ON, Canada

Full list of author information is available at the end of the article
}

(c) The Author(s). 2019 Open Access This article is distributed under the terms of the Creative Commons Attribution 4.0 International License (http://creativecommons.org/licenses/by/4.0/), which permits unrestricted use, distribution, and reproduction in any medium, provided you give appropriate credit to the original author(s) and the source, provide a link to the Creative Commons license, and indicate if changes were made. The Creative Commons Public Domain Dedication waiver (http://creativecommons.org/publicdomain/zero/1.0/) applies to the data made available in this article, unless otherwise stated. 


\section{Background}

When well implemented, antenatal care (ANC) can save lives by giving pregnant women the opportunity to receive screening for common pregnancy complications and to be referred for further diagnosis and care [1]. ANC also represents a crucial step for the promotion of healthy habits and behaviours during and after pregnancy $[1,2]$. Despite these known benefits, ANC services remain underexploited in most developing countries, including Ethiopia. According to the Ethiopian Demographic Health Survey (EDHS), in 2016, 62\% of pregnant women in Ethiopia attended ANC services at least once during their last pregnancy and 32\% of women attended four or more ANC visits [3]. Existing evidence also indicates that pregnant women in Ethiopia tend to initiate ANC late in pregnancy, with the majority of women seeking ANC services in the second or third trimester of pregnancy [3]. In addition to its key role in the diagnosis and management of pregnancy complications, ANC services are also essential for the control and prevention of infectious diseases such as malaria, that are known to greatly affect pregnancy outcomes and newborn survival [4].

In Ethiopia, the prevalence of malaria parasitemia in pregnant women is estimated to range between 2.3 and $7.1 \%$, depending on whether areas of unstable and stable endemicity are considered [5]. More recent studies have reported prevalence of malaria in pregnant women ranging between 2.8 and $44.5 \%$ [6]. Comparably, the prevalence of placental malaria is estimated at 6.5 and $2.5 \%$ in areas of relatively high and low transmission, respectively [5]. Current malaria control and prevention strategies applied by the Ethiopian Federal Ministry of Health include the use of insecticide-treated nets (ITNs) and indoor residual spraying (IRS) in households located in high transmission settings [7]. Given the relatively low prevalence of malaria in Ethiopia, intermittent preventive treatment in pregnancy (IPTp) is not part of the National Malaria Control Strategy (NMCS) [8]. Pregnant women and mothers are targeted during national ITN distribution and IRS campaigns, and health extension workers (HEWs) and other ANC service provision staff are required to distribute an ITN to every pregnant woman attending ANC [7, 9]. ANC service providers and HEWs are also expected to engage in a discussion about ITN possession and use at all visits, and discuss the negative effects that malaria can have on pregnancy [9]. Women who do not attend ANC services may therefore be at a greater risk of malaria and its associated consequences, given their limited access to ITNs and information regarding the relationship between malaria and adverse pregnancy complications.

Few studies have assessed variations in the coverage and usage of ITNs by pregnant women in relation to ANC attendance. A study from Nigeria highlights that attendance to ANC was significantly associated with ownership of bed nets [10]. However, they did not observe any difference in the use of ITNs among women who received ANC and those who did not [10]. Another study in Gabon identified that bed net coverage did not differ according to the number of ANC visits and was not associated with ANC attendance [11]. The lack of relationship was attributed to poor bed net availability within health facilities, and the absence of routine ITN distribution to pregnant women on their first visit [11]. To our knowledge, no published evidence is available on the link between ANC attendance and use of ITNs among pregnant women in Ethiopia.

To attain better maternal health outcomes, it is essential to identify barriers to and facilitators of maternal health services uptake. Herein, we assess the level and determinants of ANC attendance as well as ITN ownership and use within three rural districts of Ethiopia, and examine the relationship between ITN ownership and use, and self-reported malaria infection in pregnant women.

\section{Methods \\ Study design}

For the purpose of this study, we used data from a community-based cross-sectional survey that was conducted from October 2016 to January 2017 as part of the baseline evaluation of a larger cluster-randomized controlled trial to address barriers to safe motherhood options in Jimma Zone, Ethiopia (ClinicalTrials.gov identifier: NCT03299491), as described by Ouedraogo et al. (2019) [12]. Only women who had a pregnancy outcome (i.e. live birth, stillbirth, assisted abortion, miscarriage) in the year preceding the survey were eligible to participate.

It was determined that 24 primary health care units (PHCUs) or clusters, and a total of 3840 women would be required for the trial's baseline evaluation. The sample size calculation for the trial was based on detecting a $17 \%$ difference in the primary outcome (skilled birth attendance) between the intervention and control arms. A two-stage sampling strategy was used. We first randomly selected 24 primary health care units (PHCUs) or clusters from the 26 available in the three study districts (Gomma, Kersa, and Seka Chekorsa). From each PHCU catchment area, we then randomly selected 160 eligible women to achieve the target sample size of 3840 . Eligible women were randomly selected for face-to-face interviews using a random number generator after conducting a listing exercise with HEWs, village leaders and members of the health development army.

We sought informed consent for each woman after explaining the purpose of the survey and the risks and benefits associated with participation. Of the eligible women, $1.5 \%$ women $(N=56)$ refused to participate. No 
replacement was made for women who refused to participate. Trained interviewers used computer tablets to administer the questionnaire in the local language at the woman's household. If no woman was available for the interview on the first attempt, the household was revisited on a later date. If no respondent was available after two attempts, the household was replaced by a randomly selected alternate. Survey questions ascertained socio-demographic characteristics of the participants and their maternal health services usage before, during and after pregnancy.

\section{Study setting}

The survey was conducted in three Woredas or districts of Jimma Zone: Gomma, Kersa, and Seka Chekorsa. Jimma Zone is situated in Oromiya region, approximately $7 \mathrm{~h}$ west by road from Ethiopia's capital Addis Ababa. Oromiya is one of the regions in Ethiopia where the utilization of maternal and child health services remains suboptimal. The three districts in the study area had a population of approximately 260,000 in 2016. Maternal health services are provided at 26 health centers and 110 rural health posts located in these districts. Nationally, the 2016 EDHS reported that 48\% of women who had a live birth in the 5 years before the survey did not attend ANC services [3]. Skilled birth attendance among the 2016 EDHS participants was also low, with only $20 \%$ of live births in the 5 years preceding the survey taking place in a health facility [3]. According to the 2015 Ethiopia National Malaria Indicator Survey, 58\% of households had at least one ITN and $42 \%$ of pregnant women slept under an ITN in Oromiya region [13].

The primary malaria species of epidemiological importance in Ethiopia are Plasmodium falciparum and $P$. vivax, accounting for approximately 70 and $30 \%$ of the malaria cases, respectively [7]. The risk of malaria infection depends on the altitude, with the greatest risk of infection occurring below $2000 \mathrm{~m}$ [7]. In Gomma district, the altitude ranges from 1380 to $1680 \mathrm{~m}$, compared to $1740-2660 \mathrm{~m}$ in Kersa district and 1580-2560 m in Seka Chekorsa [14]. The three chosen areas have similar climatic conditions, with a main rainy season occurring from June to August [14]. A short rainy season is also observable in February and March [14]. The incidence of malaria infection is typically greatest during both the rainy seasons while lower infection is observed during the rest of the year [7].

\section{Study variables}

To ascertain ANC attendance in the three districts, women were asked whether they attended ANC during their last pregnancy and how many times they visited a health facility for ANC. Participants who reported not attending ANC were asked why they did not attend. To determine ITN ownership and utilization among the recruited sample, women were asked whether their household owned any ITN during their last pregnancy and how frequently they slept under an ITN during their last pregnancy (never, sometimes, often, always). We re-categorized the utilization of ITN to capture women who always used an ITN during last pregnancy and those who did not always use an ITN (never, sometimes and often). Finally, women were asked to report whether they were diagnosed with malaria during their last pregnancy.

We considered the following socio-demographic variables: age (15-18 years, 19-24 years, 25-34 years, and 35-49 years), marital status (not married, married), ethnic group (Oromo, Amhara, Others), employment status (not employed, self-employed, employed), level of education (no education, primary, secondary, higher), decision-making about health care (husband or family member, self, jointly with husband), exposure to different media sources (not at all, at least once a week, more than once a week), frequency of contact with HEWs (not at all, less than once a month, once or more times a month), household size $(\leq 4,5-8, \geq 9$ household members), children in the household $(\leq 3,4-6, \geq 7)$, reproductive history (i.e. total number of live births, miscarriages, stillbirths, and neonatal death), and last pregnancy intendedness. For employment status, the 'not employed' category included individuals who identified as housewives, students and unemployed s, while those identifying as farmers were categorized as selfemployed. Following the steps defined by the DHS group, we used a principal component analysis (PCA) to construct a household wealth index combining households' ownership of various durable assets (e.g. electricity, radio, television, refrigerator, mobile phone)), housing construction (type of materials used for floor, roof and exterior wall), type of toilet facilities, sources of water supply, and type of fuel used for cooking [15]. PCA is a statistical method that reduces a specified number of variables into a smaller number of dimensions or principal components [16]. Briefly, PCA allowed us to extract a principal component from our selected variables as a measure of socio-economic status and derive factor scores for the considered variables. Using the factor scores as weights for each variable, an overall score for each household was subsequently obtained, which can be interpreted as the household-specific wealth score. Based on their wealth score, the households were then divided into five equal quintiles (poorest, poorer, middle, wealthier, wealthiest).

\section{Statistical analysis}

All the data were synchronized to a cloud server using Open Data Kit and we exported CSV files for data management and analysis. We performed all analyses using SAS statistical software version 9.4. We assessed the 
distribution of each variable through frequency tables. We used chi-square and Fisher's exact tests, as appropriate, to explore associations between the variables and determine differences in proportions. Differences in means were assessed using t-tests. We considered a two-sided $p$-value of 0.05 as the level of statistical significance.

Using unadjusted and adjusted logistic regression models, we generated odds ratio (ORs) and corresponding 95\% confidence intervals to identify significant predictors of first ANC visit attendance. We considered all the aforementioned socio-demographic characteristics (i.e. age, marital status, employment status, level of education, decision-making about health care, exposure to television and radio, frequency of contact with HEWs, reproductive history, and last pregnancy intendedness) as potential predictors of ANC attendance in univariable analyses, and retained all significant variables $(p>0.05)$ in the multivariable models. We conducted a sub-group analysis among women who attended any ANC visit, to identify whether the participants who attended at least four ANC visits differed from women who attended three or fewer ANC visits with regards to their sociodemographic characteristics.

We used multivariable logistic regression analyses to assess the relationship between ANC attendance (no attendance, at least one visit) and ITN ownership and utilization. We considered age, marital and occupation status, education status, household wealth, and whether the dwelling was sprayed with insecticide in the last year as potential confounders and effect modifiers. Confounding was assessed by looking at the strength of the association between each variable, and ANC attendance and ITN ownership and utilization. Effect modification was investigated by introducing interaction terms between main predictors and potential effect modifiers into the regression models. We similarly assessed the main predictors of malaria infection during pregnancy using logistic regression models, considering socio-demographic factors, ITN utilization and IRS as potential predictors. For the analyses of ITN ownership and use, and malaria in pregnancy, we performed subgroup analyses considering only women living within the catchment area of PHCUs at an altitude below $2000 \mathrm{~m}$, where the risk of malaria is known to be higher.

Our analysis considered the complex cross-sectional survey sampling design, wherein women were clustered within PHCUs, as failure to do so can lead to incorrect inferences [17]. We therefore incorporated clustering of the data in all the analyses through logistic regressions with random intercept models, using the PROC GLIMMIX function in SAS 9.4. We also ran model diagnostics, including assessment of the distribution of the residuals and random effects.

\section{Results}

Characteristics of the study participants

A total of 3784 women agreed to take part in the crosssectional survey (Table 1). The response rate was of $98.5 \%$. Half of the participating women across the three districts were aged between 25 and 34 years (50.5\%). Oromo was the most dominant ethnic group, representing $94 \%$ of participants across all districts. Nearly all of the participating women were married $(97.7 \%)$ and unemployed (78.2\%). Kersa district had the highest proportion of women who reported not having a formal education (67.9\%) followed by Seka Chekorsa (53.9\%) and Gomma (47.2\%). The majority of women reported making decisions regarding health care jointly with their husband (59.2\%). Kersa had the largest proportion of participants in the poorest wealth quintile (35.9\%). An important proportion of the participants were not exposed to any media sources (i.e. newspapers/magazines (93\%), radio (46\%), and/or television (88\%)). Close to $11 \%$ of the women reported being visited frequently by an HEW during their last pregnancy (defined as one or more visits per month).

Analysis of the reproductive history of the participants revealed that, on average, the number of pregnancies per woman ranged between three and four. In Gomma, 7.6\% of the women had at least one induced abortion, compared to 4\% in Kersa and 5\% in Seka Chekorsa. The proportion of women with a history of prior miscarriage in each district was low. Across the three districts, $4.5 \%$ of the women had history of at least one stillbirth.

\section{Antenatal care attendance determinants and barriers}

Of the 3784 women who participated in the survey, 84.2\% reported attending at least one ANC visit during their last pregnancy $(90.3,75.5$ and $85.2 \%$ in Gomma, Kersa and Seka Chekorsa, respectively). Overall, 47.0\% of the participants reported seeking four or more ANC visits during their pregnancy (54.2, 38.1 and $46.4 \%$ in Gomma, Kersa and Seka Chekorsa, respectively).

We observed that $16.0 \%$ of women $(N=597)$ did not attend ANC services. The main reasons for not seeking ANC were a perceived lack of necessity of ANC services (45\%) or time (14\%), far distance to the closest health center (22\%), and the absence of transportation (13\%). Other non-negligible barriers included the absence of husband's support (8\%), sickness or complications related to pregnancy $(7 \%)$, poor service within the health facility (5\%), and the unavailability of childcare (5\%). Thirty-two participants did not provide reasons for not attending ANC.

In the multivariable analysis, education (primary education AOR: 1.47, 95\% CI: 1.16-1.87, secondary or higher education AOR: 2.38, 95\% CI:1.49-3.82, compared to no formal education) and wealth (wealthiest 
Table 1 Characteristics of the women who participated in the cross-sectional survey conducted in three districts of Jimma Zone, Ethiopia, from October 2016 to January $2017^{\text {a }}$

\begin{tabular}{|c|c|c|c|c|c|c|c|}
\hline & \multirow{2}{*}{$\begin{array}{l}\text { Total } \\
(N=3784) \\
\%\end{array}$} & \multicolumn{2}{|c|}{$\begin{array}{l}\text { Gomma } \\
(N=1402)\end{array}$} & \multicolumn{2}{|c|}{$\begin{array}{l}\text { Kersa } \\
(N=1110)\end{array}$} & \multicolumn{2}{|c|}{$\begin{array}{l}\text { Seka Chekorsa } \\
(N=1272)\end{array}$} \\
\hline & & $\mathrm{N}$ & $\%$ & $\mathrm{~N}$ & $\%$ & $\mathrm{~N}$ & $\%$ \\
\hline \multicolumn{8}{|l|}{ Maternal age (years) } \\
\hline $15-18$ & 7.5 & 95 & 6.8 & 80 & 7.2 & 110 & 8.6 \\
\hline $19-24$ & 25.5 & 345 & 24.6 & 264 & 23.8 & 356 & 27.9 \\
\hline $25-34$ & 50.5 & 736 & 52.5 & 559 & 50.4 & 615 & 48.3 \\
\hline $35-49$ & 16.5 & 226 & 16.1 & 207 & 18.6 & 191 & 15.0 \\
\hline \multicolumn{8}{|l|}{ Ethnic group } \\
\hline Oromo & 93.7 & 1262 & 90.0 & 1065 & 95.9 & 1220 & 95.9 \\
\hline Amhara & 1.9 & 58 & 4.1 & 4 & 0.4 & 11 & 0.9 \\
\hline Other & 4.3 & 82 & 5.8 & 41 & 3.7 & 41 & 3.2 \\
\hline \multicolumn{8}{|l|}{ Education level } \\
\hline No education & 55.5 & 661 & 47.2 & 754 & 67.9 & 686 & 53.9 \\
\hline Primary $^{\mathrm{b}}$ & 31.0 & 470 & 33.5 & 273 & 24.6 & 430 & 33.8 \\
\hline Secondary ${ }^{c}$ & 12.7 & 259 & 18.5 & 77 & 6.9 & 146 & 11.5 \\
\hline Higher & 0.7 & 12 & 0.9 & 6 & 0.5 & 10 & 0.8 \\
\hline \multicolumn{8}{|l|}{ Marital Status } \\
\hline Married & 97.7 & 1348 & 96.2 & 1098 & 98.9 & 1252 & 98.5 \\
\hline Not married & 2.3 & 54 & 3.8 & 12 & 1.1 & 19 & 1.5 \\
\hline \multicolumn{8}{|l|}{ Employment status } \\
\hline Not Employed ${ }^{d}$ & 78.2 & 1132 & 80.7 & 845 & 76.1 & 981 & 77.1 \\
\hline Self-employed ${ }^{e}$ & 19.2 & 211 & 15.1 & 255 & 22.9 & 260 & 20.4 \\
\hline Employed & 2.6 & 59 & 4.2 & 10 & 0.9 & 31 & 2.4 \\
\hline \multicolumn{8}{|l|}{ Wealth } \\
\hline Poorest & 20.0 & 89 & 6.3 & 399 & 35.9 & 270 & 21.2 \\
\hline Poorer & 21.3 & 252 & 17.9 & 227 & 20.4 & 326 & 25.6 \\
\hline Middle & 18.6 & 222 & 15.8 & 213 & 19.2 & 269 & 21.1 \\
\hline Wealthier & 20.1 & 309 & 22.0 & 186 & 16.7 & 265 & 20.8 \\
\hline Wealthiest & 20.0 & 530 & 37.8 & 85 & 7.66 & 142 & 11.2 \\
\hline \multicolumn{8}{|c|}{ Frequency of reading newspaper/ magazine } \\
\hline Not at all & 92.8 & 1268 & 90.4 & 1065 & 95.9 & 1180 & 92.7 \\
\hline At least once a week & 5.8 & 114 & 8.1 & 35 & 3.1 & 73 & 5.7 \\
\hline More than once a week & 1.3 & 20 & 1.4 & 10 & 0.9 & 19 & 1.5 \\
\hline \multicolumn{8}{|c|}{ Frequency of listening to the radio } \\
\hline Not at all & 45.6 & 622 & 44.4 & 534 & 48.1 & 569 & 44.7 \\
\hline At least once a week & 26.5 & 422 & 30.1 & 272 & 24.5 & 309 & 24.3 \\
\hline More than once a week & 27.9 & 358 & 25.5 & 304 & 27.4 & 394 & 30.9 \\
\hline \multicolumn{8}{|l|}{ Frequency of watching the TV } \\
\hline Not at all & 88.8 & 1070 & 76.3 & 1066 & 96.0 & 1226 & 96.4 \\
\hline At least once a week & 5.2 & 150 & 10.7 & 26 & 2.3 & 22 & 1.7 \\
\hline More than once a week & 5.9 & 182 & 12.9 & 18 & 1.6 & 24 & 1.9 \\
\hline \multicolumn{8}{|c|}{ Frequency of contact with HEW } \\
\hline Not at all & 66.4 & 856 & 61.0 & 856 & 77.1 & 802 & 63.0 \\
\hline Less than once a month & 23.0 & 348 & 24.8 & 200 & 18.0 & 323 & 25.4 \\
\hline
\end{tabular}


Table 1 Characteristics of the women who participated in the cross-sectional survey conducted in three districts of Jimma Zone, Ethiopia, from October 2016 to January $2017^{\text {a }}$ (Continued)

\begin{tabular}{|c|c|c|c|c|c|c|c|}
\hline & \multirow{2}{*}{$\begin{array}{l}\text { Total } \\
(N=3784) \\
\%\end{array}$} & \multicolumn{2}{|l|}{$\begin{array}{l}\text { Gomma } \\
(N=1402)\end{array}$} & \multicolumn{2}{|l|}{$\begin{array}{l}\text { Kersa } \\
(N=1110)\end{array}$} & \multicolumn{2}{|c|}{$\begin{array}{l}\text { Seka Chekorsa } \\
(N=1272)\end{array}$} \\
\hline & & $\mathrm{N}$ & $\%$ & $\mathrm{~N}$ & $\%$ & $\bar{N}$ & $\%$ \\
\hline One or more times a month & 10.5 & 198 & 14.1 & 54 & 4.8 & 147 & 11.5 \\
\hline \multicolumn{8}{|l|}{ Decision Making about healthcare } \\
\hline Self & 12.2 & 170 & 12.1 & 120 & 10.8 & 171 & 13.4 \\
\hline Jointly & 59.2 & 883 & 62.9 & 628 & 56.6 & 728 & 57.2 \\
\hline Husband & 27.9 & 336 & 23.9 & 355 & 31.9 & 366 & 28.7 \\
\hline Family member & 0.7 & 13 & 0.9 & 7 & 0.6 & 7 & 0.2 \\
\hline \multicolumn{8}{|l|}{ Household size } \\
\hline$\leq 4$ & 34.0 & 504 & 35.9 & 329 & 29.6 & 454 & 36.7 \\
\hline $5-8$ & 56.2 & 794 & 56.6 & 629 & 56.7 & 702 & 55.2 \\
\hline$\geq 9$ & 9.8 & 104 & 7.4 & 152 & 13.7 & 116 & 9.1 \\
\hline \multicolumn{8}{|c|}{ Number of children per household } \\
\hline$\leq 3$ & 56.2 & 848 & 60.5 & 553 & 49.8 & 725 & 57.0 \\
\hline $4-6$ & 38.7 & 506 & 36.1 & 465 & 41.9 & 493 & 38.7 \\
\hline$\geq 7$ & 5.1 & 48 & 3.4 & 92 & 8.3 & 54 & 4.25 \\
\hline \multicolumn{8}{|l|}{ Reproductive history } \\
\hline Gravidity $(\text { mean } \pm S D)^{f}$ & $3.70( \pm 2.34)$ & $3.51( \pm 2.25)$ & & $4.15( \pm 2.47)$ & & $3.52( \pm 2.27)$ & \\
\hline \multicolumn{8}{|l|}{ Live birth } \\
\hline 1 & 1.6 & 32 & 2.3 & 7 & 0.6 & 21 & 1.6 \\
\hline$>1$ & 98.4 & 1370 & 97.7 & 1103 & 99.4 & 1251 & 98.3 \\
\hline \multicolumn{8}{|l|}{ Induced abortion } \\
\hline 0 & 94.1 & 1295 & 92.4 & 1058 & 95.3 & 1210 & 95.1 \\
\hline$\geq 1$ & 5.9 & 107 & 7.6 & 52 & 4.7 & 62 & 4.9 \\
\hline \multicolumn{8}{|l|}{ Miscarriage } \\
\hline 0 & 97.4 & 1347 & 96.1 & 1088 & 98.0 & 1250 & 98.3 \\
\hline$\geq 1$ & 2.6 & 55 & 3.9 & 22 & 2.0 & 22 & 1.7 \\
\hline \multicolumn{8}{|l|}{ Stillbirth } \\
\hline 0 & 95.5 & 1314 & 93.7 & 1066 & 96.0 & 1234 & 97.0 \\
\hline$\geq 1$ & 4.5 & 88 & 6.3 & 44 & 3.9 & 38 & 2.9 \\
\hline \multicolumn{8}{|l|}{ Malaria } \\
\hline \multicolumn{8}{|l|}{ Malaria infection during pregnancy } \\
\hline Yes & 1.4 & 28 & 2.0 & 9 & 0.8 & 18 & 1.4 \\
\hline No & 98.5 & 1374 & 98.0 & 1101 & 99.2 & 1254 & 98.6 \\
\hline \multicolumn{8}{|l|}{ Bed net ownership } \\
\hline Yes & 47.6 & 874 & 62.3 & 484 & 43.6 & 444 & 34.9 \\
\hline No & 52.4 & 528 & 37.6 & 626 & 56.4 & 828 & 65.1 \\
\hline \multicolumn{8}{|l|}{ Bed net use } \\
\hline Never & 53.3 & 547 & 39.0 & 635 & 57.2 & 833 & 65.6 \\
\hline Sometimes & 8.5 & 153 & 10.9 & 83 & 7.5 & 85 & 6.7 \\
\hline Often & 11.8 & 182 & 13.0 & 147 & 13.2 & 119 & 9.4 \\
\hline Always & 26.4 & 520 & 37.1 & 245 & 22.1 & 232 & 18.3 \\
\hline
\end{tabular}

Notes: ${ }^{a}$ Numbers are rounded and may not add up to exactly $100 \% ;{ }^{b}$ considers women who completed all or some primary school years; ${ }^{c}$ considers women who completed all or some secondary school years; ${ }^{d}$ the unemployed category contains women who were unemployed, housewives or students; ${ }^{\mathrm{c}}$ the selfemployed category contains women who are farmers and/or traders; ${ }^{f}$ Defined as the number of times a woman has been pregnant 
quintile AOR: 4.29, 95\% CI: 2.89-6.35, compared to poorest quintile) were identified as the most important predictors of attending at least one ANC visit (Table 2). Frequent contacts with an HEW resulted in higher odds of ANC attendance. Similarly, women who were able to make decisions regarding their healthcare by themselves or jointly with their partners, women who experienced health problems during their last pregnancy, and women who had wanted their last pregnancy were 26, 58 and $68 \%$ more likely to attend at least one ANC visit, respectively. However, women who were of an ethnic group other than Oromo or Ahmara were 42 less likely to attend at least one ANC visit compared to women who were part of the largest ethnic group (i.e. Oromo). A significant positive association was found between frequent television use and ANC attendance; however, this was subject to small sample sizes.

Respondents had higher odds of attending four or more ANC visits if they had completed some secondary (AOR: 1.90, 95\% CI: 1.46-2.47) or higher (AOR: 5.24, 95\% CI: 1.53-17.95) education, relative to those who reported no education completion (Table 3). Wealth was also identified as an important determinant of attending four or more ANC visits, although only the odds ratios for respondents that were from the wealthiest quintile were significantly different from the poorest group (AOR:1.64, 95\% CI:1.27-2.12). Women who made decisions about their health by themselves were 33\% more likely to attend four or more ANC visits, while women who decided jointly with their partner were $32 \%$ more likely to seek four or more ANC visits. Women who were part of a household with more than seven children were $46 \%$ less likely to seek ANC four or more times than women who had three or fewer children part of their household. Desiring the last pregnancy was also a significant predictor, with an AOR of 1.37 (95\% CI: 1.17-1.62). However, we found that women who had a prior stillbirth were less likely to attend four or more ANC visits.

\section{Malaria infection and insecticide-treated net ownership and utilization during pregnancy}

We observed low rates of malaria infection during pregnancy in our sampled group with $2.0 \%(N=28), 0.8 \%$ $(N=9)$, and $1.4 \%(N=18)$ of women reporting they were diagnosed with malaria during their last pregnancy in Gomma, Kersa, and Seka Chekorsa, respectively. Fiftyone percent of the malaria cases were reported by women in Gomma. The difference in malaria prevalence across the three districts was not statistically significant. Out of the total sample, 58\% $(N=2195)$ of survey respondents were from areas below $2000 \mathrm{~m}$. Seventy-one percent of reported malaria cases were detected in women from areas at an altitude below $2000 \mathrm{~m}$. The prevalence of self-reported malaria in women living in areas below $2000 \mathrm{~m}$ was $1.9 \%(N=18)$ in Gomma, $1.0 \%$ $(N=8)$ in Kersa and 2.5\% $(N=12)$ in Seka Chekorsa (See Additional file 1: Table S1). The difference in malaria infection rates between the districts was not statistically significant $(p=0.096)$.

ITN ownership varied across the three districts with $62.3 \%$ of surveyed women in Gomma, $43.6 \%$ in Kersa, and $34.9 \%$ in Seka Chekorsa reporting that they possessed an ITN during their last pregnancy. ITN use was below the level of ITN ownership with $37.1 \%$ of women in Gomma, $22.1 \%$ in Kersa and $18.3 \%$ reportingto have always used their ITN. Considering women from areas where malaria is potentially endemic, we found that 75.5\% in Gomma, 56.9\% in Kersa and 60.2\%in Seka Chekorsa owned a ITN during their last pregnancy. The utilization of ITNs was lower with 45.4, 29.1 and 31.1\% of women who owned at least one ITNreported to have always used it during their last pregnancy in Gomma, Kersa and Seka Chekorsa, respectively (See Additional file 1: Table S1).

\section{Antenatal care attendance and insecticide-treated net ownership and use}

After adjusting for the main potential confounders and considering effect modifiers such as maternal age, ethnicity, education level, occupation status, wealth, household size, and IRS, we found that participants who attended at least one ANC had 2.07 (95\% CI: 1.62-2.63) times the odds of having owned an ITN during their last pregnancy (Table 4). Similarly, we found that women who attended at least one ANC visit were 73\% (AOR: 1.73 (95\% CI: 1.32-2.27) more likely to have always used an ITN during their last pregnancy (Table 5). Our subgroup analyses considering only the women from areas where malaria is potentially endemic (altitude below 2000 $\mathrm{m})$ suggested that women who attended at least one ANC visit were 2.41 (95\% CI: $1.82-3.19)$ times more likely to own a net and 1.75 (1.29-2.38) times more likely to report always having slept under a net during their last pregnancy (See Additional file 1: Tables S2 \& S3).

\section{Risk factors of malaria infection in pregnancy}

Women who reported that they always used an ITN during their last pregnancy were less likely to report experiencing a malaria infection, although the association was not statistically significant (AOR: 0.87 (95\% CI: 0.44-1.72)) (Table 6). High maternal age (25-49 years compared to younger age groups) and primary education level (compared to no education) were significantly associated with lower odds of malaria infection during pregnancy. Women who were self-employed were 2.8 times more likely to have a malaria infection during their past pregnancy than women who were unemployed. In the 
Table 2 Association between maternal characteristics and attending at least one antenatal care visit among women who experienced a pregnancy outcome in the past year in three districts of Jimma Zone, Ethiopia, 2016 ${ }^{a}$

\begin{tabular}{|c|c|c|c|c|c|}
\hline & $\begin{array}{l}\text { Attended at least } \\
\text { one ANC visit } \\
\text { N (\%) }\end{array}$ & $\begin{array}{l}\text { Did not } \\
\text { attend ANC } \\
N(\%)\end{array}$ & $\begin{array}{l}P \text { value for the overall } \\
\text { difference among } \\
\text { the categories }\end{array}$ & $\begin{array}{l}\text { Factors associated with } \\
\text { attending at least one } \\
\text { antenatal care visit, } \\
\text { univariate analysis } \\
\text { OR ( } 95 \% \text { Cl) }\end{array}$ & $\begin{array}{l}\text { Factors associated with } \\
\text { attending at least one } \\
\text { antenatal care visit, } \\
\text { multivariable analysis } \\
\text { AOR }(95 \% \mathrm{Cl})^{\mathrm{g}}\end{array}$ \\
\hline \multicolumn{6}{|l|}{ Maternal age (years) } \\
\hline $15-18$ & $231(7.2)$ & $54(9.0)$ & \multirow[t]{4}{*}{$<0.0001$} & Reference & Reference \\
\hline $19-24$ & $864(27.1)$ & $101(16.9)$ & & $1.83(1.25-2.67)$ & $1.27(0.84-1.91)$ \\
\hline $25-34$ & $1594(50.0)$ & 316 (52.9) & & $1.01(0.72-1.42)$ & $1.26(0.85-1.88)$ \\
\hline $35-49$ & 498 (15.6) & $126(21.1)$ & & $0.884(0.58-1.23)$ & $1.33(0.84-2.10)$ \\
\hline \multicolumn{6}{|l|}{ Ethnic group } \\
\hline Oromo & 2995 (93.9) & $552(92.5)$ & \multirow[t]{3}{*}{0.0113} & Reference & Reference \\
\hline Amhara & $66(2.1)$ & $7(1.2)$ & & $1.11(0.49-2.50)$ & $0.85(0.35-1.95)$ \\
\hline Other & $126(3.9)$ & $38(6.4)$ & & $0.56(0.37-0.84)$ & $0.58(0.38-0.89)$ \\
\hline \multicolumn{6}{|l|}{ Education level } \\
\hline No education & $1660(52.1)$ & 441 (73.8) & \multirow[t]{3}{*}{$<0.0001$} & Reference & Reference \\
\hline Primary ${ }^{\mathrm{b}}$ & $1041(32.6)$ & $132(22.1)$ & & $1.92(1.55-2.39)$ & $1.47(1.16-1.87)$ \\
\hline Secondary $^{c}$ or Higher ${ }^{d}$ & $486(15.2)$ & $24(4.0)$ & & $4.57(2.96-7.05)$ & $2.38(1.49-3.82)$ \\
\hline \multicolumn{6}{|l|}{ Employment Status } \\
\hline Not Employed ${ }^{e}$ & $2510(78.7)$ & $448(75.0)$ & \multirow[t]{3}{*}{0.0004} & Reference & Reference \\
\hline Self-employed ${ }^{f}$ & $583(18.3)$ & $143(23.9)$ & & $0.78(0.63-0.97)$ & $0.79(0.63-1.01)$ \\
\hline Employed & $94(2.9)$ & $6(1.0)$ & & $2.74(1.16-6.45)$ & $1.68(0.69-4.09)$ \\
\hline \multicolumn{6}{|l|}{ Wealth } \\
\hline Poorest & $544(17.1)$ & $214(35.8)$ & \multirow[t]{5}{*}{0.0001} & Reference & Reference \\
\hline Poorer & 649 (20.4) & $156(26.1)$ & & $1.45(1.13-1.87)$ & $1.53(1.17-1.99)$ \\
\hline Middle & $614(19.3)$ & $90(15.1)$ & & $2.319(1.74-3.08)$ & $2.52(1.87-3.39)$ \\
\hline Wealthier & $665(20.8)$ & $95(15.9)$ & & $2.27(1.71-3.03)$ & $2.37(1.76-3.19)$ \\
\hline Wealthiest & $715(22.4)$ & $42(7.0)$ & & $4.96(3.39-7.23)$ & $4.29(2.89-6.35)$ \\
\hline \multicolumn{6}{|c|}{ Frequency of reading newspaper/ magazine } \\
\hline Not at all & $2936(92.1)$ & $577(96.6)$ & \multirow[t]{3}{*}{0.0006} & Reference & Reference \\
\hline At least once a week & $203(6.4)$ & $19(3.2)$ & & $1.84(1.12-3.00)$ & $0.67(0.38-1.18)$ \\
\hline More than once a week & $48(1.51)$ & $1(0.2)$ & & $11.64(1.58-85.81)$ & $2.48(0.31-19.56)$ \\
\hline \multicolumn{6}{|l|}{ Frequency of listening to the radio } \\
\hline Not at all & $1373(43.1)$ & $352(58.9)$ & \multirow[t]{3}{*}{$<0.0001$} & Reference & Reference \\
\hline At least once a week & $879(27.6)$ & $124(20.8)$ & & $1.76(1.39-2.22)$ & $1.27(0.99-1.64)$ \\
\hline More than once a week & $935(29.3)$ & $121(20.3)$ & & $1.97(1.56-2.49)$ & $1.28(1.00-1.66)$ \\
\hline \multicolumn{6}{|l|}{ Frequency of watching the TV } \\
\hline Not at all & $2783(87.3)$ & $579(96.9)$ & \multirow[t]{3}{*}{$<0.0001$} & Reference & Reference \\
\hline At least once a week & $185(5.8)$ & $13(2.2)$ & & $2.30(1.29-4.21)$ & $1.39(0.74-2.62)$ \\
\hline More than once a week & $219(6.9)$ & $5(0.8)$ & & $6.18(2.50-15.28)$ & $2.98(1.15-7.74)$ \\
\hline \multicolumn{6}{|l|}{ Frequency of contact with HEW } \\
\hline Not at all & $2053(64.4)$ & $461(77.2)$ & \multirow[t]{3}{*}{$<0.0001$} & Reference & Reference \\
\hline Less than once a month & $773(24.2)$ & $98(16.4)$ & & $1.61(1.26-2.05)$ & $1.69(1.15-2.48)$ \\
\hline One or more times a month & $361(11.3)$ & $38(6.4)$ & & $1.86(1.29-2.67)$ & $1.66(1.27-2.13)$ \\
\hline \multicolumn{6}{|l|}{ Decision-making about healthcare } \\
\hline Husband or family member & $874(23.1)$ & $210(5.5)$ & 0.0086 & Reference & Reference \\
\hline
\end{tabular}


Table 2 Association between maternal characteristics and attending at least one antenatal care visit among women who experienced a pregnancy outcome in the past year in three districts of Jimma Zone, Ethiopia, 2016 ${ }^{\text {a }}$ (Continued)

\begin{tabular}{|c|c|c|c|c|c|}
\hline & $\begin{array}{l}\text { Attended at least } \\
\text { one ANC visit } \\
\mathrm{N}(\%)\end{array}$ & $\begin{array}{l}\text { Did not } \\
\text { attend ANC } \\
N(\%)\end{array}$ & $\begin{array}{l}P \text { value for the overall } \\
\text { difference among } \\
\text { the categories }\end{array}$ & $\begin{array}{l}\text { Factors associated with } \\
\text { attending at least one } \\
\text { antenatal care visit, } \\
\text { univariate analysis } \\
\text { OR }(95 \% \mathrm{Cl})\end{array}$ & $\begin{array}{l}\text { Factors associated with } \\
\text { attending at least one } \\
\text { antenatal care visit, } \\
\text { multivariable analysis } \\
\text { AOR }(95 \% \mathrm{Cl})^{9}\end{array}$ \\
\hline Self & $385(12.1)$ & $76(12.7)$ & & $1.16(0.86-1.57)$ & $1.10(0.81-1.51)$ \\
\hline Jointly with husband & $1928(60.5)$ & $311(52.1)$ & & $1.45(1.21-1.81)$ & $1.26(1.02-1.56)$ \\
\hline \multicolumn{6}{|l|}{ Household size } \\
\hline$\leq 4$ & $1155(36.2)$ & $132(22.1)$ & $<0.0001$ & Reference & Reference \\
\hline $5-8$ & $1751(54.9)$ & $374(62.6)$ & & $0.54(0.44-0.67)$ & $0.87(0.63-1.19)$ \\
\hline$\geq 9$ & $281(8.8)$ & $91(15.2)$ & & $0.43(0.31-0.58)$ & $0.75(0.45-1.26)$ \\
\hline \multicolumn{6}{|c|}{ Number of children per household } \\
\hline$\leq 3$ & $1882(59.0)$ & $244(40.9)$ & $<0.0001$ & Reference & Reference \\
\hline $4-6$ & $1159(36.4)$ & $305(51.1)$ & & $0.51(0.42-0.62)$ & $0.74(0.55-1.00)$ \\
\hline$\geq 7$ & $146(4.6)$ & $48(8.0)$ & & $0.47(0.33-0.68)$ & $0.76(0.41-1.39)$ \\
\hline \multicolumn{6}{|c|}{ Health problems during last pregnancy } \\
\hline No & $2491(78.2)$ & $509(85.2)$ & 0.0050 & Reference & Reference \\
\hline Yes & $694(21.8)$ & $88(14.7)$ & & $1.57(1.22-2.02)$ & $1.58(1.22-2.05)$ \\
\hline \multicolumn{6}{|l|}{ Number of live birth } \\
\hline $0-1$ & $742(23.7)$ & 79 (13.4) & $<0.0001$ & Reference & Reference \\
\hline $2-4$ & $1424(45.4)$ & $229(38.8)$ & & $0.67(0.52-0.88)$ & $0.97(0.70-1.36)$ \\
\hline$\geq 5$ & $968(30.9)$ & $282(47.8)$ & & $0.40(0.31-0.53)$ & $0.84(0.55-1.29)$ \\
\hline \multicolumn{6}{|l|}{ Number of child-deaths } \\
\hline None & $2734(85.8)$ & $485(82.1)$ & 0.0059 & Reference & Reference \\
\hline$\geq 1$ & $453(14.2)$ & $112(18.8)$ & & $0.78(0.61-0.98)$ & $1.04(0.79-1.35)$ \\
\hline \multicolumn{6}{|l|}{ Intended pregnancy } \\
\hline No & $967(30.3)$ & $309(51.7)$ & $<0.0001$ & Reference & Reference \\
\hline Yes & $2220(69.6)$ & $288(48.2)$ & & $2.18(1.81-2.63)$ & $1.68(1.38-2.06)$ \\
\hline
\end{tabular}

Abbreviations: $\mathrm{Cl}$ Confidence interval, OR Odds ratio, AOR Adjusted odds ratio

Notes: ${ }^{a}$ Numbers are rounded and may not add up to exactly $100 \% ;{ }^{b}$ considers women who completed all or some primary school years; ${ }^{c}$ considers women who completed all or some secondary school years; ${ }^{\mathrm{d}}$ secondary and higher education were combined given model conversion issues; ${ }^{\mathrm{e}}$ the unemployed category contains women who were unemployed, housewives or students; ${ }^{f}$ the self-employed category contains women who are farmers and/or traders; ${ }^{g}$ AOR and corresponding $95 \% \mathrm{Cl}$ were obtained from a multivariate model, including variables with a significant univariate test at $p$-value cut-off point of 0.05 Bold text indicates significant results at the level of $p$-value $<0.05$

analyses with women living in areas where malaria is potentially endemic, we found that employment was significantly associated with self-reported malaria infection. Compared to unemployed women, self-employed and employed women had higher odds of malaria infection (See Additional file 1: Table S4).

\section{Discussion}

Using data from a cross-sectional survey conducted with women who had a pregnancy outcome in the year preceding the survey in three rural districts of Jimma Zone, this study identified the levels and determinants of ANC attendance, the ownership and use of ITNs for the prevention of malaria in pregnancy, and the prevalence of malaria in pregnancy in the three districts.
Across the three districts, an estimated $84 \%$ of women reported attending at least one ANC visit. Our findings are similar to what Abosse et al. observed in Hadiya Zone, southern Ethiopia (86\%), but higher than what was reported in other studies conducted in eastern and northern parts of Ethiopia [18-22]. Our findings are also higher than the 51\% reported in the 2016 EDHS for Oromiya region [3, 7]. Geographical differences in ANC attendance may depend on physical proximity of communities to ANC services, and women's cultural beliefs and socio-economic status. Access to ANC services may also represent a challenge if the road conditions are poor or if the distance to the nearest health facility presents a barrier. In contrast to the high proportion of women who attended at least one ANC visit, only $47 \%$ reported attending four or more ANC visits. This is higher than 
Table 3 Association between maternal characteristics and attending four antenatal care visits among women who experienced a pregnancy outcome in the past year in three districts of Jimma Zone, Ethiopia, 2016

\begin{tabular}{|c|c|c|c|c|c|}
\hline & $\begin{array}{l}\text { Attended four antenatal } \\
\text { care visits } \\
N(\%)\end{array}$ & $\begin{array}{l}\text { Did not attend four } \\
\text { antenatal care visits } \\
\mathrm{N}(\%)\end{array}$ & $\begin{array}{l}P \text { value for the overall } \\
\text { difference among } \\
\text { the categories }\end{array}$ & $\begin{array}{l}\text { Factors associated with } \\
\text { attending four antenatal } \\
\text { care visits, univariate analysis } \\
\text { OR }(95 \% \mathrm{Cl})\end{array}$ & $\begin{array}{l}\text { Factors associated with } \\
\text { attending four antenatal } \\
\text { care visits, multivariable analysis } \\
\text { AOR }(95 \% \text { Cl) })^{\text {d }}\end{array}$ \\
\hline \multicolumn{6}{|c|}{ Maternal age (years) } \\
\hline $15-18$ & $123(6.9)$ & $108(7.6)$ & 0.0037 & Reference & Reference \\
\hline $19-24$ & $530(29.8)$ & 334 (23.6) & & $1.41(1.05-1.89)$ & $1.24(0.91-1.68)$ \\
\hline $25-34$ & $863(48.6)$ & $731(51.7)$ & & $1.01(0.78-1.36)$ & $1.04(0.76-1.42)$ \\
\hline $35-49$ & 259 (14.6) & 239 (16.9) & & $0.96(0.69-1.31)$ & $1.01(0.69-1.46)$ \\
\hline \multicolumn{6}{|l|}{ Education level } \\
\hline $\begin{array}{l}\text { No } \\
\text { education }\end{array}$ & $854(48.1)$ & $806(57.1)$ & $<0.0001$ & Reference & Reference \\
\hline Primary $^{\mathrm{b}}$ & $564(31.8)$ & 477 (33.8) & & $1.11(0.95-1.30)$ & $0.99(0.83-1.18)$ \\
\hline Secondary ${ }^{e}$ & $332(18.7)$ & $126(8.9)$ & & $2.47(1.96-3.11)$ & $1.90(1.46-2.47)$ \\
\hline Higher & $25(1.4)$ & $3(0.2)$ & & $8.38(2.50-28.07)$ & $5.24(1.53-17.95)$ \\
\hline \multicolumn{6}{|l|}{ Wealth } \\
\hline Poorest & $268(15.1)$ & $276(19.5)$ & $<0.0001$ & Reference & Reference \\
\hline Poorer & 312 (17.6) & 337 (23.8) & & $0.92(0.73-1.16)$ & $0.92(0.73-1.17)$ \\
\hline Middle & $344(19.4)$ & $270(19.1)$ & & $1.27(1.00-1.61)$ & $1.27(0.99-1.61)$ \\
\hline Wealthier & $371(20.9)$ & $294(20.8)$ & & $1.26(1.00-1.59)$ & $1.19(0.94-1.51)$ \\
\hline Wealthiest & $480(27.0)$ & $235(16.6)$ & & $2.01(1.58-2.56)$ & $1.64(1.27-2.12)$ \\
\hline \multicolumn{6}{|c|}{ Frequency of reading newspaper/ magazine } \\
\hline Not at all & $1618(91.1)$ & $1318(93.3)$ & 0.0278 & Reference & Reference \\
\hline $\begin{array}{l}\text { At least } \\
\text { once a } \\
\text { week }\end{array}$ & $128(7.2)$ & $75(5.3)$ & & $1.37(1.01-1.84)$ & $0.76(0.54-1.07)$ \\
\hline $\begin{array}{l}\text { More than } \\
\text { once a } \\
\text { week }\end{array}$ & $29(1.6)$ & $19(1.3)$ & & $1.28(0.71-2.32)$ & $0.38(0.18-0.75)$ \\
\hline \multicolumn{6}{|c|}{ Frequency of watching the TV } \\
\hline Not at all & $1490(83.9)$ & $1293(91.6)$ & $<0.0001$ & Reference & Reference \\
\hline $\begin{array}{l}\text { At least } \\
\text { once a } \\
\text { week }\end{array}$ & $133(7.5)$ & $52(3.7)$ & & $2.15(1.53-3.00)$ & $1.60(1.12-2.30)$ \\
\hline $\begin{array}{l}\text { More than } \\
\text { once a } \\
\text { week }\end{array}$ & $152(8.5)$ & $67(4.7)$ & & $1.91(1.41-2.59)$ & $1.25(0.88-1.79)$ \\
\hline \multicolumn{6}{|c|}{ Frequency of contact with HEW } \\
\hline Not at all & $1098(61.8)$ & $955(67.6)$ & 0.0027 & Reference & Reference \\
\hline $\begin{array}{l}\text { Less than } \\
\text { once a } \\
\text { month }\end{array}$ & $483(27.2)$ & $290(20.5)$ & & $1.42(1.19-1.68)$ & $1.37(1.15-1.64)$ \\
\hline $\begin{array}{l}\text { One or } \\
\text { more times } \\
\text { a month }\end{array}$ & $194(10.9)$ & $167(11.8)$ & & $0.95(0.76-1.20)$ & $0.88(0.69-1.11)$ \\
\hline \multicolumn{6}{|c|}{ Decision-making about healthcare } \\
\hline $\begin{array}{l}\text { Husband or } \\
\text { family } \\
\text { member }\end{array}$ & $426(24.0)$ & $448(31.7)$ & 0.0002 & Reference & Reference \\
\hline Self & $221(12.4)$ & 164 (11.6) & & 1.39 (1.09-1.77) & $1.33(1.03-1.70)$ \\
\hline Jointly & $1128(63.5)$ & $800(56.6)$ & & $1.46(1.24-1.72)$ & $1.32(1.11-1.55)$ \\
\hline
\end{tabular}


Table 3 Association between maternal characteristics and attending four antenatal care visits among women who experienced a pregnancy outcome in the past year in three districts of Jimma Zone, Ethiopia, 2016 (Continued)

\begin{tabular}{|c|c|c|c|c|c|}
\hline & $\begin{array}{l}\text { Attended four antenatal } \\
\text { care visits } \\
\mathrm{N}(\%)\end{array}$ & $\begin{array}{l}\text { Did not attend four } \\
\text { antenatal care visits } \\
\text { N (\%) }\end{array}$ & $\begin{array}{l}P \text { value for the overall } \\
\text { difference among } \\
\text { the categories }\end{array}$ & $\begin{array}{l}\text { Factors associated with } \\
\text { attending four antenatal } \\
\text { care visits, univariate analysis } \\
\text { OR }(95 \% \mathrm{Cl})\end{array}$ & $\begin{array}{l}\text { Factors associated with } \\
\text { attending four antenatal } \\
\text { care visits, multivariable analysis } \\
\text { AOR }(95 \% \mathrm{Cl})^{\mathrm{d}}\end{array}$ \\
\hline \multicolumn{6}{|c|}{ Household size } \\
\hline$\leq 4$ & $692(38.9)$ & $463(32.8)$ & 0.0021 & Reference & Reference \\
\hline $5-8$ & $936(52.7)$ & $815(57.7)$ & & $0.77(0.66-0.89)$ & $0.94(0.76-1.16)$ \\
\hline$\geq 9$ & $147(8.3)$ & $134(9.5)$ & & $0.74(0.57-0.97)$ & $1.24(0.81-1.92)$ \\
\hline \multicolumn{6}{|c|}{ Number of children per household } \\
\hline$\leq 3$ & $1097(61.8)$ & 785 (55.6) & 0.0009 & Reference & Reference \\
\hline $4-6$ & $612(34.5)$ & $547(38.7)$ & & $0.80(0.69-0.93)$ & $0.91(0.72-1.14)$ \\
\hline$\geq 7$ & $66(3.7)$ & $80(5.6)$ & & $0.61(0.43-0.85)$ & $0.54(0.31-0.92)$ \\
\hline \multicolumn{6}{|c|}{ Health problems during last pregnancy } \\
\hline No & $1368(77.1)$ & $1125(79.7)$ & 0.0385 & Reference & Reference \\
\hline Yes & $407(22.9)$ & $287(20.3)$ & & $1.18(1.00-1.41)$ & $1.17(0.98-1.40)$ \\
\hline \multicolumn{6}{|c|}{ Number of live birth } \\
\hline $0-1$ & $479(26.9)$ & $316(22.4)$ & 0.0147 & Reference & Reference \\
\hline $2-4$ & 789 (44.4) & 635 (44.9) & & $0.73(0.59-0.88)$ & $1.08(0.87-1.36)$ \\
\hline$\geq 5$ & $507(28.5)$ & 461 (32.6) & & $0.81(0.68-0.97)$ & $1.29(0.87-1.36)$ \\
\hline \multicolumn{6}{|c|}{ Prior stillbirth } \\
\hline No & 1708 (96.2) & $1330(94.2)$ & 0.0217 & Reference & Reference \\
\hline Yes & $67(3.8)$ & $82(5.8)$ & & $0.63(0.45-0.88)$ & $0.65(0.46-0.92)$ \\
\hline \multicolumn{6}{|c|}{ Intended pregnancy } \\
\hline No & $467(26.3)$ & $500(35.4)$ & $<0.0001$ & Reference & Reference \\
\hline Yes & 1308 (73.7) & $912(64.6)$ & & $1.54(1.32-1.79)$ & $1.37(1.17-1.62)$ \\
\hline
\end{tabular}

Abbreviations: $\mathrm{Cl}$ Confidence interval, $O R$ Odds ratio, $A O R$ Adjusted odds ratio

Notes: ${ }^{a}$ Numbers are rounded and may not add up to exactly $100 \% ;{ }^{b}$ considers women who completed all or some primary school years; ${ }^{c}$ considers women who completed all or some secondary school year; ${ }^{\mathrm{d}}$ AOR and corresponding $95 \% \mathrm{Cl}$ were obtained from a multivariate model, including variables with a significant univariate test at $p$-value cut-off point of 0.05

Bold text indicates significant results at the level of $p$-value $<0.05$

the national-level estimate of $32 \%$ reported by the DHS group [3]. In a study conducted in Jimma Zone a decade ago, about $76 \%$ of the women attended one ANC visit, while $6.5 \%$ attended the recommended four visits [23]. A more recent survey conducted in 6 districts of Jimma Zone highlighted that $93 \%$ of women attended ANC services at least once during their last pregnancy and 47\% completed four ANC visits [24]. While clear improvements over time in the coverage of ANC attendance are observed in Jimma Zone, close to $16 \%$ of our sampled women did not seek ANC at all. Of that group of $16,50 \%$ of the respondents believed that ANC was unnecessary. Although not assessed in this study, the low proportion of women seeking four or more ANC visits may be explained by women's poor satisfaction with ANC services. A qualitative assessment performed

Table 4 Association between antenatal care attendance and the ownership of insecticide-treated nets among women who experienced a pregnancy outcome in the past year in three districts of Jimma Zone, Ethiopia, 2016

\begin{tabular}{|c|c|c|c|c|}
\hline $\begin{array}{l}\text { Owned a net } \\
N(\%)\end{array}$ & Did not own a net N (\%) & $\begin{array}{l}P \text { value for the overall difference } \\
\text { among the categories }\end{array}$ & $\begin{array}{l}\text { Association with owning } \\
\text { an ITN univariate analysis } \\
\text { OR (95\% Cl) }\end{array}$ & $\begin{array}{l}\text { Association with owning } \\
\text { an ITN, multivariable analysis } \\
\text { AOR }\left(95 \% \text { Cl) }{ }^{a}\right.\end{array}$ \\
\hline 177 (9.8) & $420(21.2)$ & $<0.0001$ & Reference & Reference \\
\hline $1625(90.2)$ & $1562(78.8)$ & & $2.13(1.68-2.68)$ & $2.07(1.62-2.63)$ \\
\hline
\end{tabular}

bbreviations: ANC Antenatal care, CI Confidence interval, ITN Insecticide-treated net, OR Odds ratio, AOR Adjusted odds ratio

${ }^{a}$ Adjusting for main confounders: maternal age, ethnicity, education level, occupation status, wealth, household size and indoor residual spraying

Bold text indicates significant results at the level of $p$-value $<0.05$ 
Table 5 Association between antenatal care attendance and the utilization of insecticide-treated nets among women who experienced a pregnancy outcome in the past year in three districts of Jimma Zone, Ethiopia, 2016

\begin{tabular}{|c|c|c|c|c|c|}
\hline & $\begin{array}{l}\text { Always } \\
\text { used a net } \\
\text { N (\%) }\end{array}$ & $\begin{array}{l}\text { Did not always } \\
\text { use a net N (\%) }\end{array}$ & $\begin{array}{l}P \text { value for the overall difference } \\
\text { among the categories }\end{array}$ & $\begin{array}{l}\text { Association with always using an } \\
\text { ITN, univariate analysis } \\
\text { OR ( } 95 \% \text { CI) }\end{array}$ & $\begin{array}{l}\text { Association with always using an } \\
\text { ITN, multivariable analysis } \\
\text { AOR }(95 \% \text { CI) })^{\mathrm{a}}\end{array}$ \\
\hline \multicolumn{6}{|c|}{ ANC attendance } \\
\hline None & $85(8.5)$ & $512(18.4)$ & $<0.0001$ & Reference & Reference \\
\hline $\begin{array}{l}\text { At } \\
\text { least } \\
\text { once }\end{array}$ & $912(91.5)$ & 2275 (81.6) & & $1.86(1.43-2.43)$ & $1.73(1.32-2.27)$ \\
\hline
\end{tabular}

Abbreviations: ANC Antenatal care, Cl Confidence interval, ITN Insecticide-treated net, OR Odds ratio, AOR Adjusted odds ratio

a Adjusting for main confounders: maternal age, ethnicity, education level, occupation status, wealth, household size, indoor residual spraying

Bold text indicates significant results at the level of $p$-value $<0.05$

in Jimma Zone reported that women are unsatisfied with the services received due to a lack of confidence in the providers (healthcare workers, medical students), overcrowding of facilities and long wait time [25]. Attending fewer than four ANC visits could also be explained by the fact that women sought ANC services at an advance stage in their pregnancy and delivered before completing four ANC visits. The majority of the women in our sample attended ANC services in their second trimester (83\%). This pattern is similar to research reporting that

Table 6 Association between maternal characteristics and malaria infection during last pregnancy among women who experienced a pregnancy outcome in the past year in three districts of Jimma Zone, Ethiopia, 2016

\begin{tabular}{|c|c|c|c|c|c|}
\hline & $\begin{array}{l}\text { Malaria } \\
\text { infection during } \\
\text { last pregnancy } \\
\mathrm{N}(\%)\end{array}$ & $\begin{array}{l}\text { No malaria } \\
\text { infection during } \\
\text { last pregnancy } \\
\mathrm{N}(\%)\end{array}$ & $\begin{array}{l}P \text { value for the overall } \\
\text { difference among the } \\
\text { categories }\end{array}$ & $\begin{array}{l}\text { Factors associated with malaria } \\
\text { infection during last pregnancy, } \\
\text { univariate analysis } \\
\text { OR }(95 \% \text { Cl) }\end{array}$ & $\begin{array}{l}\text { Factors associated with malaria } \\
\text { infection during last pregnancy, } \\
\text { multivariable analysis } \\
\text { AOR }(95 \% \mathrm{Cl})^{\mathrm{g}}\end{array}$ \\
\hline \multicolumn{6}{|c|}{ Maternal age (years) } \\
\hline $15-18$ & $11(20.0)$ & $274(7.3)$ & 0.0088 & Reference & Reference \\
\hline $19-24$ & $14(25.4)$ & $951(25.5)$ & & $0.36(0.16-0.80)$ & $0.48(0.19-1.18)$ \\
\hline $25-34$ & $23(41.8)$ & 1887 (50.6) & & $0.29(0.14-0.62)$ & $0.25(0.11-0.58)$ \\
\hline $35-49$ & $7(12.7)$ & $617(16.5)$ & & $0.28(0.11-0.73)$ & $0.21(0.07-0.65)$ \\
\hline \multicolumn{6}{|c|}{ Employment status } \\
\hline $\begin{array}{l}\text { Not } \\
\text { Employed }^{b}\end{array}$ & $31(56.4)$ & $2927(78.5)$ & 0.0007 & Reference & Reference \\
\hline $\begin{array}{l}\text { Self- } \\
\text { employed }\end{array}$ & $21(38.2)$ & 705 (18.9) & & $2.97(1.68-5.22)$ & $2.81(1.55-5.11)$ \\
\hline Employed & $3(5.4)$ & $97(2.6)$ & & $2.99(0.87-10.17)$ & $3.50(0.99-12.38)$ \\
\hline \multicolumn{6}{|l|}{ Education level } \\
\hline $\begin{array}{l}\text { No } \\
\text { education }\end{array}$ & $39(70.9)$ & $2062(55.3)$ & 0.0790 & Reference & Reference \\
\hline Primary $^{\mathrm{d}}$ & $11(20.0)$ & $1162(31.2)$ & & $0.48(0.24-0.95)$ & $0.42(0.20-0.88)$ \\
\hline $\begin{array}{l}\text { Secondary } \\
\text { or higher }\end{array}$ & $5(9.1)$ & $505(13.5)$ & & $0.48(0.19-1.25)$ & $0.42(0.15-1.21)$ \\
\hline \multicolumn{6}{|c|}{ ITN utilization during pregnancy } \\
\hline No & $42(76.3)$ & $2745(73.6)$ & 0.6999 & Reference & Reference \\
\hline Yes & $13(23.6)$ & $984(26.4)$ & & $0.83(0.43-1.58)$ & $0.87(0.44-1.72)$ \\
\hline \multicolumn{6}{|c|}{ Indoor Residual Spraying } \\
\hline No & $39(70.9)$ & $2784(74.6)$ & 0.5260 & Reference & Reference \\
\hline Yes & $16(29.1)$ & 945 (25.3) & & $1.17(0.62-2.23)$ & $1.45(0.73-2.90)$ \\
\hline
\end{tabular}

Abbreviations: $\mathrm{Cl}$ Confidence interval, $O R$ Odds ratio, $A O R$ Adjusted odds ratio

Notes: ${ }^{a}$ Numbers are rounded and may not add up to exactly $100 \% ;{ }^{b}$ the unemployed category contains women who were unemployed, housewives or students; ${ }^{c}$ the self-employed category includes women who are farmers and/or traders; ${ }^{d}$ considers women who completed all or some primary school years; ${ }^{e}$ considers women who completed all or some secondary school years; ${ }^{f}$ secondary and higher education were combined given model conversion issues; ${ }^{g}$ AOR and corresponding $95 \% \mathrm{Cl}$ were obtained from a multivariate model, including variables with a significant univariate test at $p$-value cut-off point of 0.05 Bold text indicates significant results at the level of $p$-value $<0.05$ 
Ethiopian women tend to initiate ANC services in the second trimester [23-28]. A synthesis of qualitative studies in Malawi, Kenya and Ghana suggested that reproductive uncertainties, interaction with health staff and direct and indirect cost of ANC may influence the timing of ANC initiation [29]. Another qualitative assessment conducted in Ethiopia highlighted that although women are aware of when to receive ANC services, many will not seek ANC until they can feel the baby move [30]. Our findings indicate that governmental and programmatic efforts are still required to improve women's knowledge of the importance and benefits of ANC services, and to surmount barriers that prevent women to access ANC services, such as distance, road conditions, and lack of transport, and cost of transport.

Acknowledging that women who attend the recommended number of ANC visits are more inclined to deliver in the presence of a skilled birth attendant, seek postnatal care and have better pregnancy outcomes [1], we assessed the determinants of receiving four or more ANC visits. Education level and wealth status were positively associated with attending four or more ANC visits. Women who were able to make decisions about their healthcare by themselves or with their male partners were also more likely to seek ANC services four or more times during their last pregnancy. The results obtained in our analysis are in line with past studies [6, 20-22], and suggest that additional work and initiatives are needed to promote a more equitable access to ANC services, and to overcome financial and knowledge barriers to not seeking ANC services among the poorest and uneducated women.

The proportion of pregnant women who owned at least one ITN in this study was lower than the $70 \%$ observed in a study conducted within the same study area in 2015 by Birhanu and colleagues [14]. Beside targeting a different study population, consisting of household heads, the difference in the proportions of households owning an ITN between the two studies may result from the fact that the other study followed a recent net distribution [14]. The utilization of ITNs, however, is quite consistent between the two studies; it is also similar to a study carried out in 2007 in 531 households of Oromiya region, 30\% of which were recruited in Jimma Zone, wherein the utilization of ITNs among pregnant women was $59 \%$ despite the fact that roughly $90 \%$ of women owned a mosquito net [31]. This suggests that the barriers to optimal ITN utilization among pregnant women living in Jimma Zone have persisted over time. Previous studies focusing on the barriers to effective malaria prevention have indicated that perceptions regarding the risk of malaria and the effectiveness of ITNs were among the most important reasons for not using a net [7]. As suggested by our subgroup analyses, the ownership of
ITNs during pregnancy was higher among women living within the catchment area of PHCUs that are at an altitude below $2000 \mathrm{~m}$, where malaria can potentially be more endemic. Interestingly, the utilization of ITNs by women who owned ITNs was very similar to the proportion estimated considering the entire sample $(60.1 \%$ vs. $59.5 \%$ in Gomma, $51 \%$ vs. $50.6 \%$ in Kersa and $51.7 \%$ vs. $52.2 \%$ in Seka Chekorsa). This suggests that, even though women from malaria-endemic areas are more likely to receive an ITN, the utilization of ITNs throughout the pregnancy remained low. There is a possibility that women from areas below $2000 \mathrm{~m}$ do not perceive malaria as a risk. Additional research could help to identify barriers to net use by women who owned an ITN.

Our study identified a significant association between a woman's ANC attendance and the ownership and usage of a ITN during her last pregnancy. This is supported by similar findings reported in Nigeria and Mali, suggesting that ANC represents an effective mean for the delivery of ITNs to pregnant women [10, 32]. The relationship between ANC attendance and owning an ITN was stronger in our sub-group analysis (considering women living at an altitude below $2000 \mathrm{~m}$ ). ANC service providers in these PHCUs may be aware of the increased risk of malaria in their community or may have been previously engaged by mass ITN distribution campaigns. The positive associations between ANC attendance and ITN ownership and utilization, which has not been previously assessed in Ethiopia, suggest that it is important to ensure that ITNs are made available to women during their ANC visits. Still, to ensure that women have access to ITNs, it is important to first address the barriers to and predictors of antenatal care attendance that were determined in this study and other assessments.

HEWs, health development army members and other healthcare providers interacting closely with pregnant women can play an important role in identifying and reaching the non-users of ANC. Being able to distinguish the differences between users of ANC from the non-users may allow HEWs and HDA members to specifically target women during their outreach activities who are less likely to seek ANC. Additional research and programmatic efforts are required to improve the physical accessibility to ANC services.

We found that the prevalence of malaria infection was low in our sample of women who were pregnant in the preceding year. While this self-reported estimate is subject to recall or reporting biases and may be affected by seasonal variations in malaria transmission, and should therefore be interpreted with caution, we found that the majority of malaria cases were observed in women from malaria-endemic areas, although malaria prevalence was not significantly different between the groups (1.4\% versus $1.7 \%$, respectively). Our results concur with the 
prevalence of $2.3 \%$ described by Newman et al. in low transmission settings, with results from Asmamaw et al., and with general trends in malaria prevalence indicated in the Malaria Indicator Surveys (MIS) for areas below $2000 \mathrm{~m}[8,33]$. The prevalence of malaria was relatively higher than what was reported in the Health Management Information System, but lower than what Nega et al. found in South Ethiopia [12, 34] . The low prevalence of malaria detected in this study also supports current national policies against the use of IPTp in Ethiopia [5, 7]. We identified few predictive factors, which may be explained by the low prevalence and/or the insufficient sample size and power to detect key risk factors for malaria infection. Even though the risk of malaria in pregnancy is lower in Ethiopia, relative to some other African countries, the risk of rapid progression to severe malaria and associated consequences among infected individuals, is relatively high, given that pregnant women in Ethiopia are less likely to acquire temporary immunity due to the low malaria parasite exposure in their lifetime. It is therefore key to continue to improve women's access to malaria control tools (ITNs and IRS) and strengthen malaria case management services [8].

One of the main strengths of our study was the use of a large sample size to assess our associations of interest. While the cross-sectional nature of the data did not allow us to explore causal relationships, we established associations that have not been assessed in the past in this specific area of Ethiopia, and reiterated some of the gaps that will need to be overcome to improve maternal health outcomes. We considered a wide range of predicting factors in our analyses. Residual confounding remains possible, however, and measurement of some indices using self-report is subject to biases as previously mentioned. Variables pertaining to women's reproductive history may be particularly subject to reporting bias. Their association with ANC attendance should be interpreted cautiously. While women who refused to participate might have been less likely to use ANC services and/or sleep under an ITN, and may have been at greater risk of malaria, given the high response rate (i.e. $98.5 \%$ ), this would not likely have substantially changed the estimates and the direction or strength of the associations.

\section{Conclusions}

Data from a community-based cross-sectional survey suggest that ANC attendance - particularly women's attendance at four or more ANC visits - and the ownership and utilization of ITNs by pregnant women could be improved in three rural districts of Jimma Zone, Ethiopia. The main factors that were positively associated with ANC attendance were higher education and wealth status, the woman's ability to make decisions regarding their own healthcare, and the intendedness of pregnancy. This work also indicates the importance of addressing physical accessibility barriers to ANC services and improving the knowledge of pregnant women and their families on the importance of attending ANC. Our finding of a strong relationship between ANC attendance and the ownership and utilization of ITNs emphasizes the importance of reducing barriers to pregnant women's attendance to ANC, not only to receive an ITN but also to be informed on its appropriate utilization. Additional work is needed to promote a more equitable access to ANC services, and to overcome financial and knowledge barriers to not seeking ANC services among the poorest and uneducated women.

\section{Supplementary information}

Supplementary information accompanies this paper at https://doi.org/10. 1186/s12889-019-7727-8.

\begin{abstract}
Additional file 1: Table S1. Subgroup analysis for rate of self-reported malaria infection rate, and ownership and use of ITNs among women who experienced a pregnancy outcome in the past year in three districts of Jimma Zone, Ethiopia, 2016². Table S2. Subgroup analysis for association between antenatal care attendance and the ownership of bed nets among women who experienced a pregnancy outcome in the past year in three districts of Jimma Zone, Ethiopia, 2016. Table S3. Subgroup anaIysis for association between antenatal care attendance and the utilization of bed nets among women who experienced a pregnancy outcome in the past year in three districts of Jimma Zone, Ethiopia, 2016. Table S4. Subgroup analyses for association between maternal characteristics and malaria infection during last pregnancy among women who experienced a pregnancy outcome in the past year in three districts of Jimma Zone, Ethiopia, 2016.
\end{abstract}

\section{Abbreviations}

ANC: Antenatal care; AOR: Adjusted odds ratio; Cl: confidence interval; DHS: Demographic Health Survey; EDHS: Ethiopian Demographic Health Survey; HEW: Health Extension Worker; IRS: Indoor residual spraying; ITN: Insecticide-treated net; OR: Odds ratio; PHCU: Primary Health Care Unit

\section{Acknowledgments}

The study team acknowledges the women who participated in the survey for their time and participation, as well as Abebe Mamo, Getachew Kiros and Shifera Asfaw for their contribution to the cross-sectional survey data collection.

\section{Authors' contributions}

$\mathrm{MK}$ and $\mathrm{MO}$ conceived the project. MO performed all data preparation and analysis. MRG assisted with the development of the statistical analysis plan and provided guidance on the methodological and statistical aspects. MK, JK, $L A, R L, S M, K H B, G B, M A, B P, M R G$ participated in the interpretation of the findings and the writing and completion of the manuscript. All authors read and approved the final version of the manuscript.

\section{Funding}

This work was carried out with grants from the Innovating for Maternal and Child Health in Africa Initiative, co-funded by Global Affairs Canada (GAC), the Canadian Institutes of Health Research (CIHR) and Canada's International Development Research Centre (IDRC); the conclusions drawn in this study do not necessarily reflect the opinions of these funding organizations.

\section{Availability of data and materials}

The datasets used and/or analyzed during the current study are available from the corresponding author on reasonable request. 


\section{Ethics approval and consent to participate}

Ethical clearance for this study was obtained from the Research Ethics and Integrity Board at the University of Ottawa and the Jimma University Research Ethics Board. Verbal informed consent was sought from all participating women. The minimum age of participants was 15 years; the need for parental consent in the case of adolescent mothers was waived by the Jimma University Research Ethics Board.

\section{Consent for publication}

Not applicable.

\section{Competing interests}

The authors declare that they have no competing interests.

\section{Author details}

${ }^{1}$ School of Epidemiology and Public Health, Faculty of Medicine, University of Ottawa, Ottawa, ON, Canada. ${ }^{2}$ Department of Health, Behavior and Society, Faculty of Public Health, Institute of Health, Jimma University, Jimma, Oromiya Region, Ethiopia. ${ }^{3}$ Jimma Zonal Health Office, Jimma Zone, Oromiya Region, Ethiopia. ${ }^{4}$ Department of Population and Family Health, Faculty of Public Health, Institute of Health, Jimma University, Jimma, Oromiya Region, Ethiopia.

Received: 10 July 2019 Accepted: 9 October 2019

\section{Published online: 04 November 2019}

\section{References}

1. Lincetto O, Mothebesoane-Anoh S, Gomez P, Munjanja S. Antenatal Care. Opportunities for Africa's newborns: Practical data, policy and programmatic support for newborn care in Africa. The Partnership for Maternal, Newborn and Child Health; 2006.

2. de Jongh T, Gurol-Urganci I, Allen E, Jiayue Zhu N, Atun R. Barriers and enablers to integrating maternal and child health services to antenatal care in low and middle income countries. BJOG An Int J Obstet Gynaecol. 2016; 123(4):549-57.

3. Central Statistical Agency (CSA), ICF. Ethiopia Demographic and Health Survey 2016: 2016. https://dhsprogram.com/pubs/pdf/PR81/PR81.pdf. Accessed 3 Sept 2017.

4. Schantz-Dunn J, Nour NM. Malaria and pregnancy: a global health perspective. Rev Obstet Gynecol. 2009;2(3):186-92.

5. Newman RD, Hailemariam A, Jimma D, Degifie A, Kebede D, Rietveld AEC, et al. Burden of malaria during pregnancy in areas of stable and unstable transmission in Ethiopia during a nonepidemic year. J Infect Dis. 2003; 187(11):1765-72.

6. Tegegne Y, Asmelash D, Ambachew S, Eshetie S, Addisu A, Jejaw ZA. The prevalence of malaria among pregnant women in Ethiopia: a systematic review and meta-analysis. J Parasitol Res. 2019;2019:1-9.

7. USAID Ethiopia. President's malaria initiative Ethiopia - malaria operational plan FY 2017. 2017. https://www.pmi.gov/docs/default-source/defaultdocument-library/malaria-operational-plans/fy17/fy-2017-ethiopia-malariaoperational-plan.pdf?sfvrsn=6. Accessed 3 Sept 2017.

8. USAID Ethiopia. President's malaria initiative Ethiopia - malaria operational plan FY 2019. 2019. https://www.pmi.gov/docs/default-source/defaultdocument-library/malaria-operational-plans/fy19/fy-2019-ethiopia-malariaoperational-plan.pdf?sfvrsn=3. Accessed 6 Sept 2019.

9. Ethiopia Federal Ministry of Health. National strategic plan for malaria prevention control and elimination in Ethiopia. 2010. http://www. nationalplanningcycles.org/sites/default/files/country_docs/Ethiopia/ ethiopia_malaria_national_strategic_plan_2011-2015_130810.pdf. Accessed 29 Aug 2016

10. Ankomah A, Adebayo SB, Arogundade ED, Anyanti J, Nwokolo E, Ladipo O, et al. Determinants of insecticide-treated net ownership and utilization among pregnant women in Nigeria. BMC Public Health. 2012;12(1):105.

11. Bouyou-Akotet MK, Mawili-Mboumba DP, Kombila M. Antenatal care visit attendance, intermittent preventive treatment and bed net use during pregnancy in Gabon. BMC Pregnancy Childbirth. 2013;13:52.

12. Ouedraogo M, Kurji J, Abebe L, Labonté R, Morankar S, Bedru KH, et al. A quality assessment of health management information system (HMIS) data for maternal and child health in Jimma zone, Ethiopia. PLoS One. 2019;14(3): e0213600.
13. Federal Democratic Republic of Ethiopia Ministry of Health, Ethiopian Public Health Institute. Ethiopia National Malaria Indicator Survey 2015. 2016. https://www.ephi.gov.et/images/pictures/download2009/MIS-2015-FinalReport-December-_2016.pdf. Accessed 13 Feb 2019.

14. Birhanu Z, Abebe L, Sudhakar M, Dissanayake G, Yihdego Y, Alemayehu $G$, et al. Access to and use gaps of insecticide-treated nets among communities in Jimma zone, southwestern Ethiopia: baseline results from malaria education interventions. BMC Public Health. 2015;15(1):1304.

15. Rutstein SO, Johnson K. DHS Comparative Report No. 6 The DHS Wealth Index. 2004. https://dhsprogram.com/pubs/pdf/cr6/cr6.pdf

16. Vyas S, Kumaranayake L. Constructing socio-economic status indices: how to use principal components analysis. Health Policy Plan. 2006;21(6):459-68.

17. Hosmer DW, Lemeshow S. Applied Logistic Regression. Edition 3 ed. New York: Wiley; 2000.

18. Abosse Z, Woldie M, Ololo S. Factors influencing antenatal care service utilization in Hadiya zone. Ethiop J Health Sci. 2010;20(2):75-82.

19. Regassa N. Antenatal and postnatal care service utilization in southern Ethiopia: a population-based study. Afr Health Sci. 2011;11(3):390-7.

20. Seifu W, Meressa B. Maternal Health Care Service Utilization and Associated Factors among Pastoral and Agro Pastoral Reproductive Age Women Residing in Jigjiga Town, Somali Regional State, Eastern Ethiopia. Bioenerg Open access. 2017;06(01):2.

21. Kifle D, Azale T, Gelaw YA, Melsew YA. Maternal health care service seeking behaviors and associated factors among women in rural Haramaya District, eastern Ethiopia: a triangulated community-based cross-sectional study. Reprod Health. 2017;14(6):96-109.

22. Tsegay Y, Gebrehiwot T, Goicolea I, Edin K, Lemma H, Sebastian MS. Determinants of antenatal and delivery care utilization in Tigray region, Ethiopia: a cross-sectional study. Int J Equity Health. 2013;12:30.

23. Fekede B. G/Mariam a. antenatal care services utilization and factors associated in Jimma town (south West Ethiopia). Ethiop Med J. 2007; 45(2):123-33.

24. Dadi LS, Berhane M, Ahmed Y, Gudina EK, Berhanu T, Kim KH, et al. Maternal and newborn health services utilization in Jimma Zone, Southwest Ethiopia: a community based cross-sectional study. BMC Pregnancy Childbirth. 2019; 19(1):178.

25. Chemir F, Alemseged F, Workneh D. Satisfaction with focused antenatal care service and associated factors among pregnant women attending focused antenatal care at health centers in Jimma town, Jimma zone, South West Ethiopia; A facility based cross-sectional study triangulated with qualitative study. BMC Res Notes. 2014;7(1):164.

26. Yaya S, Bishwajit G, Ekholuenetale M, Shah V, Kadio B, Udenigwe O. Timing and adequate attendance of antenatal care visits among women in Ethiopia. PLoS One. 2017;12(9):e0184934.

27. Wilunda C, Quaglio G, Putoto G, Takahashi R, Calia F, Abebe D, et al. Determinants of utilisation of antenatal care and skilled birth attendant at delivery in south west Shoa zone, Ethiopia: a cross sectional study. Reprod Health. 2015;12:74

28. Dulla D, Daka D, Wakgari N. Antenatal Care Utilization and Its Associated Factors among Pregnant Women in Boricha District, Southern Ethiopia. Divers Equal Heal Care. 2017;14(2):76-84.

29. Pell C, Meñaca A, Were F, Afrah NA, Chatio S, Manda-Taylor L, et al. Factors Affecting Antenatal Care Attendance: Results from Qualitative Studies in Ghana, Kenya and Malawi. PLoS One. 2013;8(1):e53747.

30. Gebremeskel F, Dibaba Y, Admassu B. Timing of first antenatal care attendance and associated factors among pregnant women in Arba Minch town and Arba Minch District, Gamo Gofa zone, South Ethiopia. J Environ Public Health. 2015;2015:971506.

31. Baume C, Menna B, Sewnet T, Zeleke B, Asefa G, Kenea M, et al. Ethiopia bednet utilization study: why some nets owned are not used. 2007. http:// pshi.fhi360.org/pdfs/2-CAB3_Ethiopia_Bednet_Utilization_Study_Why_ Some_Nets_Owned_Are_Not_Used.pdf. Accessed 23 Oct 2017.

32. Hill J, Kayentao K, Touré M, Diarwara S, Bruce J, Smedley J, et al. Effectiveness of antenatal clinics to deliver intermittent preventive treatment and insecticide treated nets for the control of malaria in pregnancy in Mali: A household survey. PLoS One. 2014;9(3):e92102.

33. Asmamaw T, Alemu A, Alemu A, Unakal C. Prevalence of malaria and HIV among pregnant women attending antenatal clinics at Felege Hiwot referral hospital and Addis Zemen health center in northwest of Ethiopia. Int J LifeSc Bt Pharm Res. 2013;2(3):2250-3137. 
34. Nega D, Dana D, Tefera T, Eshetu T, Dal-Bianco M, Koster K, et al. Prevalence and predictors of asymptomatic malaria Parasitemia among pregnant women in the rural surroundings of Arbaminch town, South Ethiopia. PLoS One. 2015;10(4):e0123630.

\section{Publisher's Note}

Springer Nature remains neutral with regard to jurisdictional claims in published maps and institutional affiliations.

Ready to submit your research? Choose BMC and benefit from:

- fast, convenient online submission

- thorough peer review by experienced researchers in your field

- rapid publication on acceptance

- support for research data, including large and complex data types

- gold Open Access which fosters wider collaboration and increased citations

- maximum visibility for your research: over $100 \mathrm{M}$ website views per year

At BMC, research is always in progress.

Learn more biomedcentral.com/submissions 